\title{
OBSTÁCULOS EPISTEMOLÓGICOS SOBRE EL CONCEPTO DE LÍMITE DE FUNCIONES EN MANUALES DE HISTORIA DE MATEMÁTICAS
}

\author{
Iran Abreu Mendes ${ }^{1}$ \\ iamendes1@gmail.com \\ Mônica Suelen Ferreira de Moraes² \\ monicamoraes@uft.edu.br \\ ${ }^{1}$ Universidade Federal do Pará (UFPA) \\ ${ }^{2}$ Universidade Federal do Tocantins (UFT)
}

Recibido: 18/12/2019 Aceptado: 16/02/2020

\section{Resumen}

Los estudios históricos muestran que el desarrollo epistemológico del cálculo diferencial e integral siguió una trayectoria larga e irregular y, en el sentido más formal, se formó a partir del siglo XVII. Actualmente, el concepto de límite se considera un concepto fundamental en la enseñanza del cálculo, ya que la base conceptual de este conocimiento tratado en los manuales de cálculo aborda este tema, parece casi siempre definido en términos del límite. En este artículo, presentamos los resultados de un estudio sobre los supuestos obstáculos epistemológicos en el desarrollo del concepto de límite a partir de la historia de los manuales de matemáticas, con miras a superarlo en el proceso de formación de estas ideas. Como ya se mencionó, el corte tomado para el análisis estará en el estudio de los obstáculos epistemológicos del límite de función en algunos manuales de historia de las matemáticas, enfocándose en los conceptos establecidos por d'Alembert, Cauchy y Weierstrass, enfatizando los aspectos dinámicos que aparecieron como un obstáculo epistemológico para formalización de este concepto estático.

Palabras clave: Historia de las matemáticas. Obstáculo epistemológico. Cálculo Límite de funciones.

\section{EPISTEMOLOGICAL OBSTACLES ON THE FUNCTION LIMIT CONCEPT IN MATHEMATICS HISTORY MANUALS}

\begin{abstract}
Historical studies show that the epistemological development of Differential and Integral Calculus followed a long, irregular trajectory and, in the most formal sense, was shaped from the 17th century. Currently, the concept of limit is considered a fundamental concept in the teaching of Calculus, since the conceptual basis of this knowledge dealt with in Calculus manuals addresses this subject, it seems almost always defined in terms of the limit. In this article, we present the results of a study on the supposed epistemological obstacles in the development of the concept of limit from the history of mathematics manuals, with a view to overcoming it in the process of forming these ideas. As already mentioned, the cut taken for analysis will be in the study of the epistemological obstacles of function limit in some history
\end{abstract}


of mathematics manuals, focusing on the concepts established by d'Alembert, Cauchy and Weierstrass, emphasizing the dynamic aspects that appeared as an epistemological obstacle to formalization of this static concept.

Keywords: History of Mathematics. Epistemological obstacle. Calculus. Function Limit.

\title{
OBSTÁCULOS EPISTEMOLÓGICOS SOBRE O CONCEITO DE LIMITE DE FUNÇÃO EM MANUAIS DE HISTÓRIA DA MATEMÁTICA
}

\begin{abstract}
Resumo
Estudos históricos mostram que o desenvolvimento epistemológico do Cálculo Diferencial e Integral seguiu uma trajetoria long, irregular e, no sentido mais formal, foi moldado a partir do século XVII. Atualmente, o conceito de limite é considerado conceito fundamental no ensino de Cálculo, visto que a base conceitual desse conhecimento tratado nos manuais de Cálculo abordam esse assunto, parece quase sempre definida em termos do limite. Neste artigo, apresentamos os resultados de um estudo sobre os supostos obstáculos epistemológicos no desenvolvimento do conceito de limite a partir dos manuais de história da matemática, com um olhar para a sua superação no processo de formação dessas ideias. Conforme já mencionado, o recorte tomado para análise será no estudo dos obstáculos epistemológicos de limite de função em alguns manuais de história da matemática, focando os conceitos estabelecidos por d'Alembert, Cauchy e Weierstrass, enfatizando os aspectos dinâmicos que figuraram como obstáculo epistemológico à formalização deste conceito estático.
\end{abstract}

Palavras-chave: História da Matemática. Obstáculo epistemológico. Cálculo. Limite de função.

\section{INTRODUÇÃO}

Este artigo é fruto de uma pesquisa mais ampla que está em desenvolvimento no Programa de Pós-Graduação em Educação em Ciências e Matemática (PPGECEM) da Rede Amazônica de Educação em Ciências e Matemática (REAMEC) em parceria com o Grupo Pesquisa sobre Práticas Socioculturais e Educação Matemática (GPSEM) da Universidade Federal do Pará (UFPA). Este trabalho se identifica com a linha de pesquisa História da Matemática em três dimensões do GPSEM, na qual desenvolve estudos e pesquisas relacionados à História da Matemática em suas dimensões epistemológicas, pedagógicas e patrimoniais.

Sabemos que nos cursos de Matemática do ensino superior há um alto índice de reprovação e desistência da disciplina de Cálculo, a qual está presente no currículo de cursos de graduação, tais como engenharia, física, administração, computação, entre outros. Sabemos 
ainda que muitas dessas dificuldades de aprendizagem estão relacionadas com o entendimento da noção de limite. Com isso, direcionamos a temática dessa pesquisa para o âmbito do Cálculo, particularmente para limite de função de uma variável real ${ }^{1}$.

Os conceitos que deram início ao desenvolvimento epistemológico do Cálculo envolvendo as relações conceituais entre limite, derivada e integral, levaram séculos para serem formulados e aceitos no pensamento matemático hegemônico ao longo da história. Vários foram os entraves desde o pensamento antigo grego de separar o discreto e o contínuo, motivado pelo "horror ao infinito", até à invenção e formalização do Cálculo como conhecemos atualmente.

Nos remetemos a esses entraves como obstáculos epistemológicos, no sentido de Bachelard (1996), pois foram conflitos que ocorreram ao longo do desenvolvimento histórico de alguns conceitos matemáticos entre o conhecimento antigo e o novo, possibilitando a criação do Cálculo. Ressaltamos, porém, que esses imaginados obstáculos epistemológicos surgidos no desenvolvimento do Cálculo foram sendo superados na medida em que os desafios epistêmicos foram vencidos a cada resposta alcançada na aventura da criação matemática. Igualmente, destamos que Bachelard (1996) institui a noção de obstáculo epistemológico no contexto do desenvolvimento da ciência, no concerne à produção de conhecimento, não para os processos de aprendizagem.

Entretanto, foi no âmbito dos processos de ensino e aprendizagem da matemática que Brousseau (1997) apresentou uma reformulação do conceito de obstáculo epistemológico, mantendo a ideia do conflito de conhecimentos de estados inferiores, que funcionavam em determinado contexto, mas que em situações de um estado superior, já se configura como um obstáculo epistemológico.

Diante desta problemática anunciamos as seguintes perguntas de pesquisa: como autores dos manuais de história da matemática tratam dos obstáculos epistemológicos e de sua superação no desenvolvimento das ideias de limite de função na perspectiva de Bachelard? Caso esteja evidenciada tal abordagem, como podemos identificar potencialidades para a superação dos obstáculos epistemológicos para o ensino de limite de função a partir da perspectiva de Brousseau?

\footnotetext{
${ }^{1}$ A função de uma variável real será tratada no decorrer desse artigo somente pelo termo função.
} 
Com base nas questões estabelecidas anteriormente, nosso objetivo é apresentar um estudo dos obstáculos epistemológicos no desenvolvimento do conceito de limite de função a partir dos manuais de história da matemática com um olhar para a sua superação no processo de formação do conceito e de que modo podemos utilizá-los para potencializar uma abordagem de ensino do tema.

Para tanto, dentre os obstáculos epistemológicos conhecidos sobre este conceito, optamos por enfatizar os aspectos dinâmicos presente na ideia de limite proposta por Newton e o discreto presente no desenvolvimento do trabalho do Leibniz, com o olhar para a superação desses entraves na formalização do conceito de limite, no sentido de Bachelard (1996). Dessa forma procuramos trazer contribuições para o ensino de Matemática, pelo enfoque na exploração epistemológica das informações históricas do conceito de limite, apontando possibilidades de superação das dificuldades conceituais e didáticos do processo em suas ações de ensino nesta temática. Para começar, precisamos primeiramente tratar sobre o que tomamos como obstáculo epistemológico.

\section{OBSTÁCULOS EPISTEMOLÓGICOS}

Inicialmente esclarecemos que tomaremos dois aspectos conceituais relativos à expressão obstáculo epistemológico, uma vez que o embasamento teórico deste artigo permeia a noção de obstáculo epistemológico apresentada por Bachelard (1996) com relação ao desenvolvimento epistemológico de conceitos científicos ao longo da história e a compreensão de Brousseau (1983; 1986; 1997), que transporta essa noção de Bachelard para o âmbito do ensino de matemática.

Segundo Bachelard (1996), a noção de obstáculo epistemológico pode ser estudada mediante o desenvolvimento histórico do pensamento científico, sendo que é ao aprofundarmos a noção de obstáculo epistemológico que conferimos pleno valor espiritual à história deste pensamento. Assim, alguns dos principais obstáculos epistemológicos abordados por Bachelard (1996), que não só causam a estagnação da construção do pensamento científico, mas também contribuem para o seu retrocesso são: experiência primeira; conhecimento geral; obstáculo verbal; conhecimento unitário e pragmático; obstáculo substancialista; obstáculo animista; obstáculos do conhecimento quantitativo. 
Com base no exposto no parágrafo anterior, é importante dar destaque aos obstáculos experiência primeira e conhecimento geral, pois para Bachelard (1996) o primeiro obstáculo a ser superado na formação do espírito científico é a experiência primeira, que não constitui uma base segura, haja vista que é colocada antes e acima da crítica, um elemento necessariamente integrante do espírito científico. A respeito do conhecimento geral como obstáculo para o conhecimento científico, Bachelard (1996) expõe que a ciência do geral é uma suspensão da experiência, um fracasso do empirismo inventivo, podendo o espírito científico se enganar ao estar atraído pelo universal.

De outro lado, Brousseau (1986; 1997), embasado em Bachelard, afirma que os obstáculos de origem epistemológica são inerentes ao saber e podem ser identificados ao longo da história da matemática, quando se observa as dificuldades que os matemáticos encontraram, para a compreensão e utilização desses conceitos, pois esse tipo de obstáculo é constitutivo do próprio conhecimento. É assim que para Brousseau (1986) um obstáculo epistemológico é sempre um conhecimento e não um estado de ausência de conhecimento. Nesse sentido, ainda que os obstáculos possam ser detectados entre as dificuldades, estas deverão ser sempre reformuladas a fim de serem caracterizadas como conhecimentos propriamente ditos. O obstáculo deve possuir tanto um domínio no qual ele se revele pertinente, válido e eficaz, como também um domínio no qual esse mesmo conhecimento se revele inadaptado, falso ou ineficaz e, portanto, uma fonte de erros. O obstáculo é um tipo de conhecimento que oferece uma resistência que deve ser identificada, atestada e explicada.

Para identificação desses obstáculos, Brousseau (1989) propõe um método de pesquisa e indica que devemos encontrar erros sistemáticos e concepções em torno das quais esses erros se agrupam. Em seguida, encontrar obstáculos na história da matemática, e confrontar os obstáculos históricos com os obstáculos que se manifestam no ato da aprendizagem.

Bachelard (1996, p. 28) afirma que "a história da matemática é maravilhosamente regular. Conhece períodos de pausa. Mas não conhece períodos de erro”, nesse sentido, para ele na matemática não há obstáculos epistemológicos. Entendemos que não há obstáculos epistemológicos na matemática porque eles vêm sendo superados ao longo do seu próprio desenvolvimento. Neste sentido, o que estamos denominando de obstáculos epistemológicos, corroborando com as ideias de Bachelard (1996), referem-se às dificuldades enfrentadas e 
superadas no desenvolvimento dos conceitos matemáticos como é o caso da noção de limite, foco deste artigo.

Sobre esse posicionamento, Asimov (1996) afirma que na matemática, diferentemente de outras ciências, não há correção significativa, só extensão. Cada matemático acrescenta algo ao que já está posto, com uma base sólida e funcional. Também entendemos que a matemática não apresenta crise no seu desenvolvimento, não há ruptura que represnete uma modificação extrema, ou seja, mudança de paradigma, no sentido de Thomas Kuhn (2011), que negue o anterior. Esse ponto de vista fortalece a premissa de que a matemática em si não apresenta obstáculos epistemológicos em conceitos já formalizados, pois para desenvolvê-lo, já ocorreu o processo de superação desses obstáculos epistemológicos.

Schubring (2018) também levanta uma reflexão sobre a existência de obstáculos epistemológicos em matemática, questionando se os números negativos podem ser tomados como exemplo de obstáculos epistemológicos. Schubring (2018) apresenta uma análise sobre o desenvolvimento histórico desses números questionando o conceito de obstáculo epistemológico dado como uma "sonolência" individual, considerando que as escolhas realizadas ao longo do desenvolvimento deste conceito foram feitas com pleno conhecimento das epistemologias concorrentes.

Nossa perspectiva de utilização do conceito de obstáculo epistemológico se aproxima da proposta de Schubring (2018) que assume que as reflexões de Bachelard (1996) podem ser usadas para esclarecer as dificuldades dentro dos desenvolvimentos conceituais. Schubring (2018) defende que precisamos estudar os conceitos matemáticos como foram desenvolvidos considerando a época e a cultura na qual se situam.

Nossa intenção não é de reduzir a história como fonte para identificar erros cometidos no passado por matemáticos ao desenvolverem determinados conceitos, mas sim, identificar obstáculos superados ao longo do desenvolvimento histórico do conceito de limite de função, mais especificamente os aspectos dinâmicos deste conceito, analisando as formas de superação ocorridas no contexto do desenvolvimento histórico, com vistas a reflextir sobre as possibilidades de tomar essas formas de superação como potencial aliado para as ações de ensino na atualidade. Cabe-nos, entretanto, verificar como tais obstáculos apareceram ao longo do desenvolvimento histórico do conceito de limite de uma função. 
Antes de adentrarmos nessa parte da discussão é preciso refletir que talvez o processo de aparecimento e superação de tais obstáculos epistemológicos tenham desafiado a curiosidade e criatividade dos matemáticos que se dedicaram a responder as questões desafiadoras que foram surgindo ao longo dos tempos e espaços. Não significa, porém, afirmar ou negar que a invenção exerceu um papel importante na criação matemática, pois embora não seja suficiente, atualmente podemos refletir e admitir que foi contributiva pelo menos no que se refere à experiência criativa e sua sistematização que conduziu à elaboração teórica de conceitos e princípios matemáticos.

Assim é possível destacar que criatividade está no coração do desenvolvimento conceitual em busca da superação desses obstáculos epistemológicos concernentes ao desenvolvimento do conceito de limite, que se mostra como um exemplo perfeito para uma discussão sobre a arte criativa na produção matemática em todos os tempos e espaços nos quais se estabelece a cultura humana. Neste sentido, a história mostra o quanto os matemáticos foram impulsionados por uma simples pergunta: e se? É realmente um momento em que uma dinâmica criativa completa pode surgir através da curiosidade, como o fez Arquimedes em seu tempo, assim como Newton, Leibniz, d'Alembert, Cauchy e Weiertrass, conforme trataremos a seguir.

Antes, porém, consideramos a relevância de um destaque sobre o que Abraham Moles (1957; 1970; 2007), estabelece em sua obra sobre o processo de criação intelectual, quando estabelece que é a partir de um ângulo filosófico e científico, que se caracteriza o conceito de criatividade como uma atitude do espírito para reorganizar os elementos do campo de percepção de uma maneira original e susceptível de dar lugar a operações em qualquier campo fenomenal, como é o caso do desenvolvimento epistemológico do Cálculo, mas específicamente, do conceito de limite de uma função.

A respeito da superação dos desafios caracterizados como obstáculo epistemológico ao desenvolvimento da Matemática, Mendes (2015) apresenta seu ciclo de problematização, imaginação, formulação e representação do conhecimento (figura 01), no qual assevera que

(...) esse conhecimento construído se origina de problematizacões estabelecidas na interacõo sociedade, cognicão e cultura, na tentativa de encontrar solucões para as situacões problemáticas surgidas no contexto. As respostas encontradas são configuradas sob dois aspectos: questões resolvidas e questões em aberto. As questões resolvidas originam-se das respostas as problematizacoões surgidas. $\mathrm{Na}$ medida em que tais respostas sao codificadas, visando a sua comunicacão e também a 
sua utilizacão na busca de respostas acerca de situacões problemáticas similares, quase sempre originam novas questões (questões em aberto). As questões em aberto, por sua vez, constituem-se em fontes provocadoras para novos estudos, transformando-se assim em um processo cíclico de producõo do conhecimento (Mendes, 2015, p.98).

Figura 01. Ciclo de problematização, imaginação, formulação e representação do conhecimento.

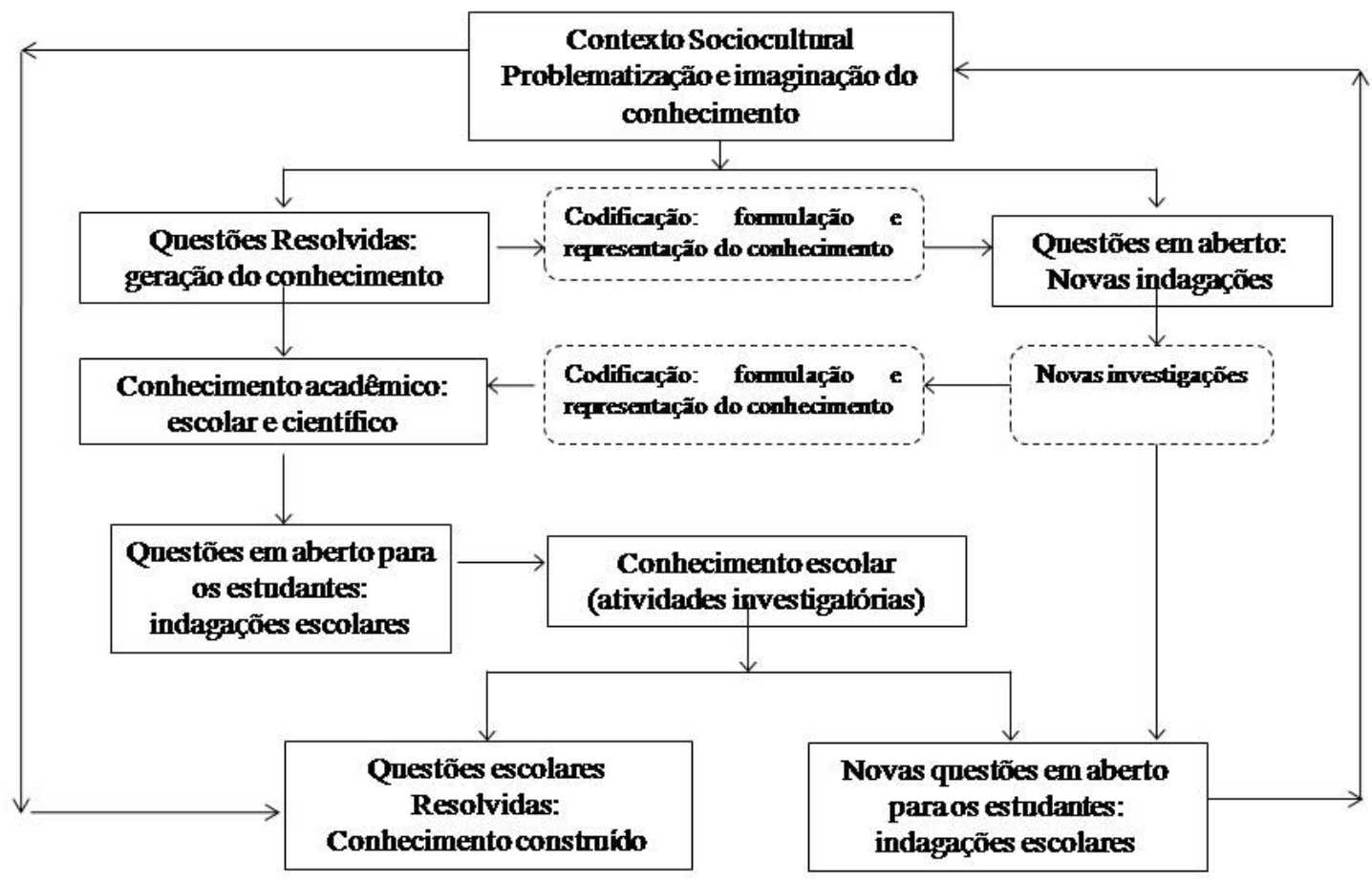

Fonte: Mendes (2015)

O ciclo proposto por Mendes (2015) explicita encaminhamentos processuais para que sejam desenvolvidas ações investigativas em história da Matemática para o ensino tomando como princípios e métodos, os movimentos de superação das dificuldades encontradas nas formulaç eos conceituais e de modo elas se apresnetam para os estudantes, conforme a organização programática da matemática escolar e de sua institucionalizaão em componentes curriculares, manuais escolares ou livros didáticos. É nesta direção que abordaremos a seguir aspectos concernentes aos obstáculos epistemológicos no desenvolvimento do conceito de limite.

\section{OBSTÁCULOS EPISTEMOLÓGICOS NO DESENVOLVIMENTO HISTÓRICO DO CONCEITO DE LIMITE DE FUNÇÃO}


Para analisar os aspectos dinâmicos do conceito de limite, com o olhar para a superação dos obstáculos epistemológicos, precisamos analisar as ideias apresentadas por matemáticos que trouxeram esse aspecto ao longo de seu desenvolvimento, bem como o de quem os ajudou a superá-lo.

Neste sentido, iniciamos examinando como a noção de limite ganhou forma a partir da “invenção" do Cálculo. Nesta seção apresentaremos, então, a noção de limite desenvolvidos nos trabalhos de Isaac Newton (1643 - 1727) e Gottfried Wilhelm Leibniz (1646 - 1716), de modo a fazer uma contextualização dos aspectos mais específicos tratados nesse processo, para em seguida, centrar-nos nas ideias de limite apresentadas por Jean Le Rond d'Alembert (1717-1783), Augustin-Louis Cauchy (1789-1857) e Karl Wieirstrass (1815 - 1897).

Dentre os manuais de História da Matemática analisados, Boyer (1996), (1992), Eves (2011), Bos (1985) e Roque (2018), somente foi apresentada a definição do conceito de limite, pós Newton e Leibniz, posta por d'Alembert, Cauchy e Weierstrass, por isso a ênfase dada para esses três matemáticos neste artigo. Os manuais consultados são obras traduzidas para o português muito utilizadas em disciplinas de História da Matemática na graduação em matemática no Brasil.

Carl Benjamim Boyer (1906 - 1976) se dedicou à História da Matemática e contribuiu para a profissionalização desse campo de saber. Boyer (1996) objetivou em sua obra "História da Matemática" apresentar esta história com fidelidade não só para com a exatidão matemática, mas também para com a perspectiva e detalhes históricos, fazendo um arranjo cronológico com mais ênfase a elementos históricos. A primeira versão, em inglês, foi publicada em 1968 e conforme a corrente dominante da época, percebemos que o foco do autor está mais na matemática do que nos matemáticos, pois, nesta altura, considerava-se que os detalhes biográficos têm menos influência no desenvolvimento dos conceitos abordados.

A obra "Cálculo" da série Tópicos de história da Matemática para uso em sala de aula, publicada originalmente em inglês em 1969, foi escrita por Boyer (1992) para auxiliar o ensino de Cálculo a partir de uma perspectiva histórica, como material didático. Apresenta uma primeira parte que descreve de modo geral a história do Cálculo e, na segunda parte, são oferecidas "cápsulas" evidenciando episódios mais específicos desta história.

A obra "Introdução à história da matemática", de Howard Eves (1911 - 2004), foi publicada 1953. Eves (2011) tenta, neste livro, introduzir a história da matemática aos alunos 
de graduação dos cursos superiores de matemática. Por este motivo, além da narrativa histórica, o autor apresenta em cada capítulo exercícios e temas a serem discutidos, com o objetivo de motivar os estudantes para a pesquisa na área. Eves (2011) escreveu seu livro como um professor de matemática com interesse em história, mantendo a organização textual cronologicamente. A obra ainda apresenta panoramas culturais escritos por seu filho, Jamie Eves, com o intuito de mostrar um aprofundamento do cenário cultural das várias eras e épocas da história da matemática.

O "Curso de história da matemática: origens e desenvolvimento do cálculo" foi publicado em 5 unidades por Margaret Eleanor Baron (?) e Henk Jan Maarten Bos (1940), em 1974, originalmente em inglês. Os autores tem por objetivo com essa obra tratar de aspectos importantes para o desenvolvimento do cálculo, tais como o conceito de integração, de diferenciação, o teorema fundamental do cálculo, o desenvolvimento de notações e símbolos, o conceito de função, de quantidades infinitamente pequenas, indivisíveis, quantidades divisíveis ad inifitum, e, por fim o abandono dos infinitésimos e a determinação do conceito de limite como o conceito fundamental do cálculo. Nos limitamos a analisar apenas a Unidade 4, que aborda os fundamentos do cálculo do século XVIII e um pequeno resumo dos principais progressos do cálculo no século XIX. Os autores têm uma preocupação pedagógica nesta obra em apresentar questões em meio aos vários capítulos presentes em cada unidade que nem sempre se limitam a consulta da obra em si. Também há a preocupação de trazer as respostas.

Em uma obra bem mais recente que as anteriores, Tatiana Roque (1970) publica, em 2018, o livro "História da matemática: uma visão crítica, desfazendo mitos e lendas", partindo dos modos como a História da Matemática já foi escrita para recontar essa história. A autora inicia cada capítulo do livro com um relato da visão convencional sobre o conteúdo em questão e em seguida, apresenta uma contextualização mais ampla que leve em conta fatores culturais ou filosóficos, explicitando as relações intrínsecas entre as práticas matemáticas e seu contexto.

\subsection{O conceito de limite por Newton e Leibniz}

Até o fim do século XVII, já se tinham feito muitas integrações, muitas cubaturas, quadraturas, processo de diferenciação e muitas tangentes a curvas haviam sido construídas, a ideia de limite já fora concebida e o teorema fundamental reconhecido no âmbito do 
desenvolvimento do Cálculo. Eves (2011), aponta que "faltava ainda a criação de um simbolismo geral com um conjunto sistemático de regras analíticas formais e também um redesenvolvimento, consistente e rigoroso, dos fundamentos da matéria” (p. 435).

Por volta de 1666, Isaac Newton (1643 - 1727) sintetizou um estudo baseado no que ele chamava de "método de fluxões", no qual as noções de movimento desempenharam um papel central e significativo. O primeiro livro no qual Newton delineia seu cálculo foi o $D e$ analysi per aequationes numero terminorum infinitas, publicado em 1711, onde o autor descreve a extensão do uso da palavra "análise", argumentando que os algoritmos matemáticos que lidam com processos infinitos são tão respeitáveis quanto aqueles que se aplicam à álgebra ordinária, concedendo assim um espaço considerável ao método das "séries infinitas". Boyer (1959) enfatiza que a contribuição de Newton está no reconhecimento de que tudo isso constitui parte de uma nova análise: a aplicação de processos infinitos ao estudo geral de funções.

Para Baron e Bos (1985), três ideias fundamentaram a invenção do Cálculo por Gottfried Wilhelm Leibniz (1646 - 1716): seu interesse pelo simbolismo e pela notação vinculada à sua ideia de uma linguagem simbólica geral; o reconhecimento de que somar sequências e tomar as suas diferenças são operações inversas e que a determinação de áreas e a de tangentes são operações inversas; e, o uso de um triângulo característico para deduzir transformações gerais de áreas.

Boyer (1959) destaca que o elemento essencial na invenção do cálculo por Leibniz, foi o reconhecimento, em 1676, de que também estava construindo uma análise nova e universal. Em seus primeiros artigos publicados, Leibniz expôs que seu novo método não apresentava impedimentos para funções irracionais ou transcendentes.

Newton e Leibniz chegaram ao Cálculo através de caminhos diferentes. Não só é diferente a linguagem com que ambos expressaram as ideias fundamentais do Cálculo, mas também em termos de concepção pode-se verificar uma diferença grande entre os seus trabalhos. Tanto Newton quanto Leibniz podem ser considerados como os primeiros a expressar a ideia da reciprocidade entre a diferencial e a integral, que constitui o Teorema Fundamental do Cálculo. Mas as maneiras de ver o Cálculo eram distintas.

A compreensão do significado dessa situação levou cada um deles a desenvolver uma linguagem, uma lógica e um simbolismo para a nova matéria, conforme explica Boyer (1992). 
No entanto, nenhum dos dois "estavam em condições de apresentar uma fundamentação lógica convincente" (p. 21). Boyer (1992) aponta que Newton chegou mais próximo disso, quando descreveu sua ideia de "primeira e última razões".

Usando notações modernas, Boyer (1959) parafraseia a formulação de Newton descrevendo $y$ como a razão das quantidades "evanescentes" $\Delta y$ e $\Delta t$. Newton esclarece que "por razão última das quantidades evanescentes deve-se entender a razão das quantidades, não antes de desaparecerem, nem depois, mas com as quais elas desaparecem” (p. 21).

Fazendo alusão aos fundamentos do novo conhecimento, Newton refere-se por vezes aos infinitesimais, outras aos limites, e ainda, à uma intuição física básica, sendo esta última abordagem a mais adotada posteriormente. Por outro lado, Leibniz e seus seguidores basearam o desenvolvimento da teoria sobre os diferenciais infinitamente pequenos, de primeira e segunda ordem.

Boyer (1959) mostra que, de modo geral, podemos dizer que Newton baseou seu Cálculo em noções de continuidade, enquanto Leibniz tomou como base a ideia discreta das mônadas. Ambas as maneiras de abordar o problema mostraram-se igualmente úteis, pois, enquanto não estava estabelecida a noção de limites, as ideias de movimento contínuo e de infinitésimos discretos surgiram como tentativas de esquematizar as primeiras impressões sensíveis quanto à variação.

Isso explica por que o Cálculo, nos estágios iniciais do seu desenvolvimento, estava cercado de conceitos de geometria do movimento, e com explicações de indivisíveis e infinitamente pequenos, pois estas ideias eram sugeridas pela intuição e experiência de continuidade.

Segundo Boyer (1996), Newton trabalhava com quantidades variáveis com um significado baseado na noção de movimento contínuo, as considerando a partir do movimento contínuo de pontos, retas e planos. Ele não considerava as variáveis como agregados de elementos infinitesimais.

Ao longo de seu trabalho, Newton fez referência aos infinitésimos, mas foi removendo até chegar a considerar que quantidades matemáticas não deveriam ser constituídas por momentos ou partes muito pequenas, mas sim como descritas pelo movimento contínuo. Newton sentia-se incomodado em interpretar suas proposições em termos de infinitesimais, 
preferindo usar velocidades, que também chamava de movimentos, mutações ou fluxões de quantidades. Assim, Newton refere-se ao seu Cálculo como o Método das Fluxões.

Já Leibniz tem outra maneira de encarar as coisas. Para Leibniz, a visualização do Cálculo se dá de forma estática, considerando as variáveis como percorrendo sequências de valores infinitamente próximos. No seu Cálculo há pouco uso de conceitos de movimento.

Conforme Baron e Bos (1985, p. 70), Leibniz entendia como necessários os infinitésimos, e construía sobre eles analogias, buscando uma visualização do Cálculo através de considerações discretas, através do diferencial: "A diferencial de uma variável y é a diferença $(d y)$ entre dois valores consecutivos de $y$ em uma sequência de números infinitamente próximos".

As concepções de Leibniz, quanto ao discreto, e a de Newton, quanto ao contínuo, recaíram na teoria do Cálculo, que posteriormente define melhor o que eram os números reais e a ideia de limite. Portanto, vemos que ambas as abordagens de Newton e Leibniz são caminhos a invenção do Cálculo.

O Cálculo foi um grande instrumento matemático descoberto no século XVII, pois se mostrou notavelmente eficiente para atacar diversos problemas não resolvidos em tempos anteriores. Com isso, diversos pesquisadores da época foram atraídos a utilizá-lo, mesmo que de modo despreocupado com os seus fundamentos, até porque para a concepção matemática da época, o rigor utilizado satisfazia. Segue-se para os séculos XVIII e XIX a tarefa de melhor fundamentar o Cálculo, conforme o que se entendia por rigor em cada época, bem como o refinamento de muitos conceitos importantes de outros ramos da matemática.

\subsection{O conceito de limite por d'Alembert, Cauchy e Weierstrass}

Apresentaremos as concepções de limite tratadas por d'Alembert, Cauchy e Weierstrass a partir de manuais de História da Matemática, Boyer (1996), (1992), Eves (2011), Bos (1985) e Roque (2018), de maneira integrada, buscando compreender como a noção de obstáculo epistemológico pode ser observada nas descrições feitas por esses autores.

O primeiro matemático a destacarmos nesta análise é Jean Le Rond d'Alembert (1717 1783). Ele considerava que a epistemologia do Cálculo estava fundada na ideia de limite. Segundo Eves (2011), para d'Alembert era preciso desenvolver uma teoria dos limites bem 
estruturada para colocar em bases firmes os fundamentos da análise. A respeito da maneira como d'Alembert concebia o conceito de limite, Thomas L. Hankins (1989) informa que

(...) na representação geométrica de quantidades infinitesimais, o diferencial desempenha um papel essencial. No entanto, quando se trata de escrever seus artigos matemáticos para a Enciclopédia, ele tem um conceito muito avançado da derivada. Nos seus artigos "Diferencial", "Fluxão" e "Limites", ele insiste que apenas existem derivadas e que os diferenciais de primeira ordem não têm significado real (Hankins, 1989, p. 188).

Para reiterar mais ainda a afirmação de Hankins (1989, idem) mencionamos a seguir um trecho do artigo “Différenttiel”, escrito por d'Alembert (Encyclopédie, IV, p. 987):

... não existem quantidades infinitamente pequenas de primeira ordem no cálculo diferencial ... As quantidades que se chama assim são xensadas divididas por outras quantidades supostamente infinitamente pequenas, e ... neste afirmam que marcam não quantidades infinitamente pequenas, nem mesmo frações cujo numerador e denominador são infinitamente pequenos, mas o limite de uma razão de duas quantidades finitas. O mesmo vale para segundas diferenças e para outras de ordem superior.

Seguindo neste mesmo raciocínio, ainda no artigo "différenttiel”, d'Alembert afirmou que "a diferenciação de equações consiste simplesmente em achar os limites da razão de diferenças finitas de duas variáveis na equação", assumindo que "uma quantidade é alguma coisa ou é nada", opondo-se ao ponto de vista de Leibniz e Euler, excluindo a noção de diferenciais como grandezas infinitamente pequenas (Boyer, 1996, p. 311).

Segundo Boyer (1996), a expressão "primeira a última razão" de Newton foi interpretada por d'Alembert como um limite em vez de uma primeira ou última razão de duas quantidades que estão apenas surgindo. Para d'Alembert,

uma grandeza é o limite de outra grandeza quando a segunda pode aproximar-se da primeira tanto quanto se queira, embora a primeira grandeza nunca possa exceder a grandeza da qual ela se aproxima; de modo que a diferença entre tal quantidade e seu limite é absolutamente indeterminável (Baron e Bos, 1985, p. 28).

Boyer (1996) apresenta essa definição da seguinte maneira: "chamou quantidade o limite de uma segunda quantidade [variável] se a segunda pode se aproximar da primeira de mais perto que por qualquer quantidade dada (sem coincidir com ela)" (p. 311). Eves (2011) não apresenta a definição de limite posta por d'Alembert. 
Esta compreensão de limite foi apresentada no verbete "Limite", de 1765, e conforme as narrativas de Bos (2018) e Roque (2018), d'Alembert afirma que a quantidade não pode ultrapassar seu limite nem atingi-lo; o limite sempre se aproxima, chegando cada vez mais perto da quantidade, mas difere sempre dela tão pouco quanto se queira.

Para Boyer (1996), a imprecisão nessa definição foi removida nas obras de matemáticos do século dezenove. Nesta última afirmação, mais uma vez Boyer (1996) não considera o ferramental matemático ou ausência dele na época de d'Alembert criticando a falta de rigor ao enunciar o seu conceito de limite.

Podemos considerar que há um obstáculo epistemológico na associação da passagem ao limite com um movimento físico, quando no momento de "aproximar-se tanto quanto se queira" ou "se aproxima chegando cada vez mais perto da quantidade", enquanto a noção de limite é entendida formalmente como "estática".

Segundo Roque (2018), d'Alembert afirmava, que o uso das quantidades infinitamente pequenas pode abreviar as demonstrações, mas que ainda assim elas não devem ser aceitas, já que é preciso deduzir as propriedades das curvas com "todo o rigor" necessário. Sem os infinitésimos, d'Alembert definiu infinitamente grande em termos de limites. Prosseguiu definindo quantidades infinitamente grandes de ordem superior de modo parecido ao utilizado atualmente ao tratarmos de ordens de infinito em relação a funções. Boyer (1996) afirma que d'Alembert negava o infinito atual, pois pensava em grandezas geométricas e não na teoria dos conjuntos proposta um século depois.

Podemos evidenciar um obstáculo epistemológico neste contexto, dado que a presença da noção de infinitamente pequeno dificultou a noção de limite, mas ao mesmo tempo o infinitesimal também foi um fator de progresso uma vez que a noção de limite foi desenvolvida em parte na reação contra o infinitamente pequeno.

Uma das desvantagens apontadas por Baron (1985) no conceito de limite de d'Alembert é a afirmação de que a variável não pode alcançar seu limite. Entendemos o pressuposto de que as variáveis são contínuas em seus domínios e não ultrapassam certos valores. Nesse sentido, o conceito de limite toca na questão da continuidade referente ao conceito de variável. Sem uma explicação mais sólida sobre isso, o conceito de limite não foi bem aceito. 
As terminologias utilizadas para conceituar limite até aqui dão a ideia de movimento no tempo, a variável deve aumentar ou diminuir com relação ao seu limite, mas não deve oscilar, nem voltar, se remetendo a uma noção geométrica da diferença entre uma grandeza variável e uma grandeza constante, se manifestando como mais um obstáculo epistemológico a ser superado posteriormente. Ao considerar as variáveis como crescentes ou decrescentes, d'Alembert remete-se à ideia clara de limite como uma fronteira, pois só pode estar situado na fronteira do domínio da variável.

Roque (2018) esclarece que o desenvolvimento das ideias fundamentais do Cálculo não se deu somente no interior da matemática. Durante os séculos XVII e XVIII, as discussões acerca de sua natureza e legitimidade dos métodos infinitesimais se inseriam em um domínio amplo que incluía além da matemática, a filosofia e a física. Bos (1985) salienta que não havia uma distinção clara entre a análise e campos da física matemática. Até neste ponto, o Cálculo lidava principalmente com variáveis, até se desenvolver a noção de função pela utilização desta noção nos diversos campos das ciências naturais, e se entender a ideia de o Cálculo operava com funções.

Faremos um breve comentário de como os autores dos manuais abordam as contribuições de Leonhard Euler (1707 - 1783) para o desenvolvimento do Cálculo, por mais que não tenha uma formulação para a noção de limite por ser determinante a conceituação e função apresentada por este matemático.

Conforme Boyer (1996), Euler tornou o cálculo diferencial e o método dos fluxos parte da "análise", o estudo de processos infinitos, com fundamental importância para a ideia de função definida por ele como "qualquer expressão analítica formada daquela quantidade variável e de números ou quantidades constantes" (p. 306), tornando o cálculo em um campo de estudos primariamente geométrico.

Não foi explicitado por Euler o que ele chamara de expressão analítica, para Bos (1985), mas pareciam incluir expressões algébricas e expressões que envolviam funções elementares transcendentais. Numa posterior explicação de Euler, chamou de função “qualquer variável que dependa de outra de tal modo que, quando a segunda varia, a primeira também varia" (Bos, 1985, p. 36).

Boyer (1996) considera ingênua a atitude de Euler somente considerar funções bem comportadas. Entendemos esse julgamento feito por Boyer (1996) advindo de um olhar do 
hoje para o passado, deixando de valorizar os feitos de Euler pelo o que havia disponível na época. Bos (1985) esclarece que Euler supunha que as funções, de modo geral, fossem bem comportadas, pois tinham as mesmas propriedades que as expressões analíticas mais comuns. Essa suposição remonta a "problemas" de continuidade e diferenciabilidade.

Conforme Bos (1985), Euler afirmou em 1755, em seu livro sobre cálculo, que quantidades infinitamente pequenas não existiam, argumentando que as quantidades menores que qualquer quantidade finita são iguais a zero. Entretanto, para Euler os zeros podem ter uma razão finita. Assumia que diferenciais são símbolos para quantidades que são zero, mas, no entanto, são qualitativamente diferentes.

Entre as abordagens de Euler, Roque (2011) observa que a operacionalização algébrica do cálculo de diferenças leibniziano é justificado com argumentos mecânicos, como o da possibilidade de dividir a matéria infinitamente, e teve uma intensa recepção no período da hegemonia do método analítico.

Bos (1985) faz uma reflexão sobre como "pensavam" os matemáticos anteriores a Cauchy sobre a fundamentação do Cálculo. Afirma que os matemáticos supunham que poderiam resolver este "problema" com um argumento profundo e inteligente, a ser posto no início dos livros de Cálculo e tudo que fora estudado estava "correto". Nesta reflexão, podemos perceber como o autor considera o conhecimento matemático anterior como um obstáculo para a produção do conhecimento novo. Bos (1985) ainda considera que o problema só seria resolvido a partir do desenvolvimento do rigor (fazendo alusão ao rigor do século XIX) na definição dos conceitos básicos da análise.

O segundo matemático a ser destacado neste trabalho é Augustin-Louis Cauchy (1789 1857) que teve como motivação para o desenvolvimento de definições mais precisas dos conceitos no âmbito do Cálculo, segundo Boyer (1996), o estudo de variáveis reais e variáveis complexas que não eram possíveis de serem representadas em duas dimensões. Roque (2018) já apresenta como motivação a preocupação didática na maneira como Cauchy propôs reorganizar a análise de modo a explicar melhor seus conceitos básicos.

O principal objetivo dos livros de Cauchy, segundo Bos (1985), seria reconciliar o rigor, da análise, com um entendimento mais simples sobre as quantidades infinitamente pequenas. 
Para Cauchy, a variável é uma quantidade numérica indeterminada que inclui todos os valores determinados, sem exceção. As variáveis de Cauchy passavam por vários valores diferentes, mas não atingiam, necessariamente, todos os valores, isto é, elas podiam ser limitadas a um dado intervalo (Roque, 2018).

De acordo com Roque (2018), Cauchy definia função a partir da distinção entre variáveis independentes e dependentes: “quando quantidades variáveis são ligadas de modo que, quando o valor de uma delas é dado, pode-se inferir os valores das outras, concebemos ordinariamente essas várias quantidades como expressas por meio de uma delas que recebe, portanto, o nome de "variável independente"” (p. 332). E as quantidades, expressas por meio da variável independente, são chamadas de funções desta variável.

Cauchy tornou fundamental o conceito de limite de d'Alembert dando-lhe um caráter aritmético mais preciso, dispensando assim a geometria e infinitésimos ou velocidades: “quando os valores sucessivamente atribuídos a uma variável se aproximam indefinidamente de um valor fixo de modo a acabar diferindo dele por tão pouco quanto se queira, este último chama-se o limite dos outros todos (Boyer, 1996, p. 355). Boyer (1992) não considera essa definição aceitável comparado ao que conhecemos como limite atualmente, por não esclarecer os papéis das variáveis depende e independente, assumindo mais uma vez o olhar para a história com referências a atualidade.

Bos (1985) chama atenção para o conceito de variável de Cauchy ainda sugerir aumento ou decréscimos contínuos, apresentando um conceito de limite muito parecido com o de d'Alembert, sem excluir a possibilidade da variável alcançar o seu limite, e se utilizando das vantagens da utilização da função e não de variáveis.

Boyer (1996) enfatiza ainda que Cauchy definiu o infinitésimo como uma variável dependente ao passo que outros o entendiam como um número fixo muito pequeno: “diz-se que uma quantidade variável se torna infinitamente pequena quando seu valor numérico diminui indefinidamente de modo a convergir ao limite zero" (p. 355), muito próximo da definição de continuidade que utilizamos hoje. Assim, os conceitos de função e limite de função eram fundamentais no Cálculo proposto por Cauchy.

Ainda sobre o infinitamente pequeno, Cauchy não considera uma quantidade infinitamente pequena como zero, nem como um quantidade constante menor do que qualquer quantidade finita, mas sim como variável que se aproxima de zero (BOS, 1985). 
Com Cauchy o limite se dissocia da ideia de quantidades infinitamente pequenas, como também fornece os elementos necessários para que essas quantidades se separem do aspecto metafísico presente até então. No entanto, conforme Boyer (1992) esta mesma noção de limite, encontra-se associado ainda a ideias intuitivas como valores sucessivos aproximar-se indefinidamente, e diferença tão pequena quanto se queira. Dessa forma, verificamos a presença de ideias de movimento que se caracterizam como obstáculo na definição de limite de Cauchy.

Percebemos um outro obstáculo epistemológico na definição de limite de Cauchy ligada a falta de simbologia adequada, pois ele não deixou clara a dependência entre a vizinhança do ponto em que se calcula o limite e o da proximidade do ponto que é o limite.

Para Roque (2018), ao procurarmos na obra de Cauchy antecedentes das noções modernas em análise, podemos nos deparar com erros que frustrarão nossas expectativas. Concordarmos com a autora ao afirmar que poderia ser mais proveitoso ver Cauchy como alguém que buscava um tipo de rigor que já não era o do século XVIII, fundado na algebrização, mas que também não era o rigor desenvolvido no século XIX.

Eves (2011) cita as contribuições de Cauchy, em 1821, para o Cálculo ao definir então continuidade, diferenciabilidade e integral definida em termos do conceito de limite, enfatizando que Cauchy não havia atingido o verdadeiro cerne das dificuldades na procura de uma fundamentação sólida para a análise, pois a teoria dos limites fora construída embasada em uma noção simples de número real. Eves (2011) não cita as definições dadas por Cauchy, nem aprofunda o assunto.

A noção de limite refere-se a funções cujos valores são pontos e não subconjuntos de um espaço topológico. O termo "diferença" que aparece de diferentes maneiras nas definições de limite deve ser entendido como determinado pela topologia do espaço. Se atualmente a ideia de limite está intimamente relacionada com a operação de fechamento topológico, a intuição geométrica, em algumas situações, influência o pensamento do mais próximo estar associado a fronteira do conjunto. Entendemos essa situação advinda de um obstáculo de cunho geométrico que só poderá ser superado com um conceito bem formado de número real.

Uma das grandes dificuldades da história do conceito de limite, compreendida também como obstáculo epistemológico, nesta altura, era de abstrair do contexto geométrico a cinemática, não para trabalhar a "grandeza", mas sim os números. A noção de limite 
precisava destes obstáculos para que pudesse passar de um estágio embrionário para outro de sua construção. $\mathrm{O}$ aparecimento do conceito geral de função foi um ponto decisivo que permitiu no século XIX uma clara articulação da noção de limite livre da intuição geométrica e física.

Com a noção de limite formulada, Karl Weierstrass (1815 - 1897) formaliza o Cálculo, introduzindo a linguagem dos Épsilons $(\varepsilon)$ e Deltas ( $\delta$ ). No tópico denominado "a aritmetização da análise”, Boyer (1996) cita as contribuições de Weierstrass para o conceito de limite. Weierstrass tentou separar o Cálculo da geometria e baseá-lo no conceito de número. Percebeu que para fazer isso, era necessário dar uma definição de número irracional independente do conceito de limite. Então, Weierstrass decidiu a questão da existência do limite de uma sequência convergente tomando a própria sequência como número ou limite, corrigindo o erro lógico de Cauchy. Boyer (1996), nesta obra, não apresenta a definição formal de limite dada por Weierstrass.

O problema básico a ser resolvido concernente ao rigor em análise, segundo Bos (1985), estava relacionado ao conceito que quantidade, assumida essencialmente como geométrico até o século XVIII. Ao retirar desse conceito todas as conotações geométricas e tomar quantidade como número real, foi possível formalizar a análise.

Boyer (1992, p. 27), nesta outra obra, evidencia a definição do conceito de limite apresentada por Weierstrass: "diz-se que $L$ é um limite da função $f(x)$ para o valor $x=a$ se, dado qualquer número positivo $\varepsilon$, existe um número positivo $\delta$ tal que $|f(x)-L|<\varepsilon$ para qualquer $x$ que verifique $0<|x-a|<\delta$. Nesta definição, é a função que tem um limite, não a variável. Percebemos que Boyer (1992) enaltece a beleza matemática desta definição, bem como a resolução das lacunas lógicas que ela, juntamente com o desenvolvimento do formalismo de outros conceitos à época resolvem no âmbito da matemática.

Assim, segundo Eves (2011), Weierstrass defendeu um programa, a aritmetização da análise, no qual o sistema dos números reais fosse rigoroso e hoje a análise pode ser deduzida logicamente de um conjunto de postulados que caracterizem o sistema dos números reais. Eves (2011) e Roque (2018) não apresentam a definição de limite de Weierstrass. Eves (2011) comenta de forma genérica a contribuição de Weierstrass para a formalização deste conceito e Roque (2018) foca no desenvolvimento do conceito de rigor desta época. 
Ao observar os modos de apresentação do desenvolvimento do conceito de limite a luz de d'Alembert, Cauchy e Wieirstrass, percebemos, principalmente em Boyer (1996, 1992) e Eves (2011) frases que remetem ao julgamento do passado com os olhos do presente. Roque (2018) é mais cuidadosa e crítica a este modo de analisar a história.

De modo geral, nesta análise percebemos o aspecto dinâmico como obstáculo epistemológico muito presente no conceito de limite até Weierstrass, que se deve, em grande parte, a aproximação dos conceitos matemáticos com outras áreas do conhecimento, que utiliza a matemática como ferramenta de interpretação de fenômenos, permanecendo, até Cauchy, a questão de saber se uma grandeza variável atingiu o seu limite ou não.

Em seguida, temos os obstáculos relacionados à dificuldade de abstrair da geometria e da cinemática os elementos necessários para se trabalhar com números. Os obstáculos epistemológicos estavam ligados à interpretação geométrica das grandezas, à interpretação dinâmica do conceito de variável, e, às interpretações metafísicas dos conceitos de continuidade. Temos ainda os obstáculos relativos à noção de função que enfatiza a concepção de contínuo, nesse contexto, de Leibniz até Cauchy.

\section{REFLEXÕES SOBRE O TEMA E DESDOBRAMENTOS FUTUROS}

Consideramos o estudo sobre o conceito de obstáculo epistemológico um desafio para o educador matemático, no sentido de se pensar sobre a natureza da matemática e como esse conhecimento se desenvolveu epistemologicamente. Talvez esse desafio seja uma das chaves de superação das dificuldades conceituais e didáticas de muitos professores de matemática em quaisquer níveis de ensino. Por essa reflexão consideramos que este tipo de desafio poderá proporcionar investimentos acadêmcios em busca de contribuições para uma compreensão mais ampla sobre o tipo de conhecimento que o professor de matemática deve desenvolver ao aceitar a tarefa de ensinar.

Neste artigo nos preocupamos em entender como as narrativas dos autores de manuais de História da Matemática nos possibilitam refletir sobre os obstáculos epistemológicos inerentes ao desenvolvimento das ideias de limite de função na perspectiva de Bachelard. Percebemos a possibilidade de refletir sobre esta temática e nos debruçamos na análise de

alguns manuais de História da Matemática muito utilizados no Brasil, Boyer (1992; 1996), Eves (2011), Bos (1985) e Roque (2018), com o objetivo de apresentar um estudo dos 
obstáculos epistemológicos no desenvolvimento do conceito de limite de função a partir dos referidos manuais com o olhar para a sua superação no processo de formação do conceito.

Embora matematicamente, toda a noção de limite esteja contida na sua definição em $\mathcal{E}$ e $\delta$, existe uma lacuna entre o conceito (no sentido intuitivo) de limite, e a definição da noção de limite. Esta lacuna é proveniente do próprio conceito e ao modo que podemos defini-lo. O aspecto dinâmico não é desenvolvido pela definição, que é estática.

Não é incomum o conceito intuitivo de limite e a sua definição formal serem compreendidas de maneira independente. É possível internalizar da definição o suficiente para lidar com a maioria dos exercícios que possamos encontrar durante um estudo a nível de graduação sobre o assunto, sem adquirir necessariamente o conceito intuitivo de limite. Por outro lado, também é possível compreender uma série de aspectos fundamentais do conceito de limite (por exemplo, aproximação), sem entender a definição em $\mathcal{\varepsilon}$ e $\delta$.

Há muita discussão sobre se considerar obstáculos epistemológicos encontrados na história de determinado conceito resistentes nos alunos da atualidade. Não temos essa interpretação determinante da utilização pedagógica do estudo dos obstáculos epistemológicos para o ensino de matemática, mas entendemos que pode ser usado para esclarecer as dificuldades dentro dos desenvolvimentos conceituais. Por isso, a preocupação, no contexto deste trabalho, de compreender como podemos identificar potencialidades para a superação dos obstáculos epistemológicos para o ensino de limite de função, a partir da perspectiva de Brousseau.

Observando os obstáculos evidenciados neste trabalho, propomos que se faça um estudo histórico do desenvolvimento desse conceito. Este estudo, orientado pelo professor, possibilita ao aluno uma visão mais ampla e localizada em termos sócio-histórico-cultural da construção do conceito de limite e dos entraves encontrados em seu desenvolvimento.

O estudo histórico pode se iniciar com a tarefa, para os estudantes, de uma pesquisa quanto ao desenvolvimento do conceito de limite. Essa tarefa pode ser dividida entre grupos de alunos, com temas pré-estabelecidos. São sugestões: Invenção do Cálculo, Cálculo de Newton, Cálculo de Leibniz, Conceito de limite de d'Alembert, Conceito de limite de d'Alembert, Formalização do conceito de limite etc. Estes temas sugeridos estão focados no estudo das definições de alguns matemáticos quanto ao conceito de limite com o objetivo de 
possibilitar uma discussão entre as concepções de limite estudados e os conceitos até então compreendidos pelos alunos.

A partir de então, sugerimos uma discussão das dificuldades que os matemáticos tiveram pra se chegar à definição formal de limite, se essas dificuldades são as mesmas que os alunos encontram nas definições construídas por eles, observando os obstáculos que precisavam ser transpostos.

As possíveis dificuldades que podem emergir nos diálogos entre os alunos, ou até mesmo podem ser provocadas por reflexões provocadas pelo professor, podem ser associadas aos obstáculos identificados na análise dos conceitos de limite de d'Alembert, Cauchy e Weierstrass. Quais as suas aproximações e diferenças com o modo que estudamos hoje? O que faltava, matematicamente, ser desenvolvido para que uma definição se aproximasse mais da outra?

Dando ênfase aos aspectos dinâmicos do conceito de limite, pode ser realizada uma discussão das características de movimento presente na noção intuitiva de limite a partir do contexto histórico do qual se originou, estabelecendo também uma discussão quanto as grandezas atingirem ou não o seu limite. Ressaltamos ainda a importância de se ater no ensino de Cálculo o método geométrico aliado ao método aritmético/algébrico, por cada um proporcionar as interpretações necessárias para a apreensão do conceito de limite.

Outra possível discussão pode ser feita em torno das dificuldades encontradas historicamente de abstrair do contexto geométrico a cinemática, não para trabalhar a "grandeza", mas sim os números, chegando assim, na definição formal de limite. O que $\varepsilon$ significa na definição de limite de função? O que $\delta$ significa na definição de limite de função? Qual o significado da relação entre $\varepsilon$ e $\delta$ na definição formal de limite de função? Por que devemos buscá-la? São perguntas que podem ser feitas nesta discussão.

Entendemos que os apontamentos dados possibilitam seguir um norte no ensino de limite a partir de "onde o aluno está", por via do diálogo dos estudantes entre os mesmos e com o professor, permitindo que as compreensões do aluno não se transformem em obstáculos para sua aprendizagem, mas sim, o propulsor para a construção de uma nova compreensão mais sólida mediada pelo professor.

Em termos de aprofundamento teórico para o professor, entendemos que o estudo dos obstáculos epistemológicos superados ao longo do desenvolvimento histórico do conceito de 
limite proporcione uma visão mais geral do Cálculo e, ao mesmo tempo, mais específica no sentido de possibilitar a criação de ferramentas didáticas mais eficientes para o ensino deste conceito.

\section{REFERENCIAS}

Asimov, I. (1996). Prefácio. In: Boyer, C. B. História da matemática. 2. ed. Trad. Elza F. Gomide. São Paulo: Editora Edgard Blücher.

Bachelard, G. (1996). A formação do espírito científico. Trad. Estela dos Santos Abreu. Rio de Janeiro: Editora Contraponto.

Baron, M. E.; BOS, Henk Jan Maarten. (1985). Newton e Leibniz (Unidade 3). Trad. Rudolf Maier. In: Baron, M. E. \& Bos, H. J. M. Curso de história da matemática: origens e desenvolvimento do cálculo. Brasília: Editora Universidade de Brasília.

Bos, H. J. M. (1985). O Cálculo do século XVIII: fundamentos (Unidade 4). Trad. José Matoso Miranda Mendes. In: Baron, M. E. \& Bos, H. J. M. Curso de história da matemática: origens e desenvolvimento do cálculo. Brasília: Editora Universidade de Brasília.

Boyer, C. B. (1959). The history of calculus and its conceptual development. New York: Dover publications.

Boyer, C. B. (1992). Cálculo. Trad. Hygino H. Domingues. v. 6. São Paulo: Editora Atual, (Tópicos de história da matemática para uso em sala de aula).

Boyer, C. B. (1996). História da matemática. Trad. Elza F. Gomide. 2. ed. São Paulo: Editora Edgard Blücher.

Brousseau, G. (1983). Les obstacles épistémologiques et les problèmes en mathématiques. Recherches en Didactique des Mathématiques. Grenoble: La Pensée Sauvage-Éditions 4(2), 164-198.

Brousseau, G (1986). Fondements et Méthodes de la Didactique des Mathématiques. Recherches en Didactique des Mathématiques. Grenoble: La Pensée Sauvage-Éditions, 7(2), 33-116.

Brousseau, G. (1989). Obstacles épistémologiques, conflits socio-cognitifs et ingénierie didactique. In: Bednarz, N., \& Garnier, C. (Eds). Construction des savoirs: obstacles et conflits. Montréal: Cirade, p. 277-285.

Brousseau, G. (1997). Theory of didactical situations in mathematics: didactique des mathématiques, 1970-1990. Mathematics Education Library: Kluwer Academic Publishers.

Eves, H. (2011). Introdução à história da matemática. Trad. Hygino H. Domingues. 5 ed. Campinas, SP: Editora da Unicamp.

Hankins, Thomas L. (1989). Jean d'Alembert: Homme de Science. In: Jean d'Alembert Savant et Philosophe: portrait à plusieurs voix. Paris: Éditions des Archives contemporaines, p. 188-205.

Mendes, I. A. (2015). História da Matemática no Ensino: entre trajetórias profissionais, epistemologias e pesquisas. São Paulo: Editora Livraria da Física. (Coleção História da matemática para Professores).

Moles, A. (2007). A criação científica. São Paulo: Perspectiva. (Coleção Estudos - Filosofia da Ciência). 
Moles, A. (1957). La création scientifique. Genève: Kister, (1 ${ }^{\mathrm{a}}$ reimpressão, 1998).

Moles, A., \& Caude, R. (1970). Créativité et méthodes d'innovation das l'entreprise. Paris: FayardMame.

Kuhn, T. S. (1962). Estrutura das Revoluções Científicas. 11. ed. Tradução Beatriz Viana Boeira e Nelson Boeira. Rio de Janeiro: Perspectiva, 2011. (Coleção Debates, 115).

Roque, T. (2018). História da matemática: uma visão crítica, desfazendo mitos e lendas. Rio de Janeiro: Editora Zahar.

Schubring, G. (2018). Os números negativos: exemplos de obstáculos epistemológicos? São Paulo: Editora Livraria da Física. (Série história da matemática para professores).

\section{Autores:}

Iran Abreu Mendes

Bolsista Produtividade em Pesquisa Nível 1C do CNPq. Pós-doutorado em Educação

Matemática pela UNESP/Rio Claro (2008). Doutorado em Educação pela Universidade Federal do Rio Grande do Norte (2001) e Mestrado em Educação pela Universidade Federal do Rio Grande do Norte (1997). Graduação em Licenciatura em Matemática pela Universidade Federal do Pará (1983). Atualmente é professor Titular do Instituto de Educação

Matemática e Científica da Universidade Federal do Pará (IEMCI), e pesquisador do Programa de Pós-graduação em Educação em Ciências e Matemáticas. Tem experiência no ensino de Cálculo, Geometria Analítica e Euclidiana, História da Matemática, História da

Educação Matemática, Didática da Matemática e Fundamentos Epistemológicos da

Matemática. Desenvolve pesquisas sobre: Epistemologia da Matemática, História da Matemática, História da Educação Matemática, História para o Ensino de Matemática, Práticas Socioculturais e Educação Matemática, Diversidade Cultural e Educação Matemática. Líder do Grupo de Pesquisa sobre Práticas Socioculturais e Educação Matemática (GPSEM/UFPA). Mais informacões no Currículo Lattes: http://Lattes.cnpq.br/4490674057492872. ORCID: https://orcid.org/0000-0001-7910-1602. URL: http://www.iranmendes.com. E-mail: iamendes1@gmail.com

Mônica Suelen Ferreira de Moraes

Mestre em Educação Matemática pelo Programa de Pós-Graduação em Educação em Ciências e Matemáticas (PPGECM/IEMCI/UFPA). Doutoranda em Educação em Ciências e Matemática pelo Programa de Pós-Graduação em Educação em Ciências e Matemática (PPGECEM) da Rede Amazônica de Educação em Ciências e Matemática (REAMEC). Graduada em Licenciatura em Matemática pela Universidade do Estado do Pará (UEPA2010). Professora da Universidade Federal do Tocantins (UFT/Arraias). Membro do Grupo de Estudos e Pesquisas em Educação Matemática na Formação de Professores (GEPEMFOR) e do Grupo de Pesquisa sobre Práticas Socioculturais e Educação Matemática (GPSEM). Desenvolve pesquisas em epistemologia da matemática, história da matemática e formação de 
professores. Mais informações no Currículo Lattes: http://Lattes.cnpq.br/8488999128970916. Orcid: https://orcid.org/0000-0001-8806-2027.E-mail: monicamoraes@uft.edu.br. 